\title{
Embolia pulmonar como hallazgo inicial de enfermedad de Graves
}

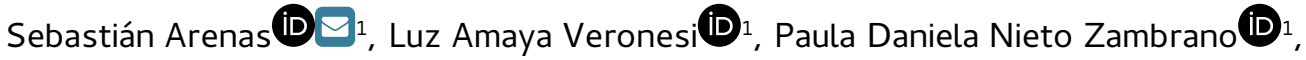 \\ Dayann Estefany Mendieta Roa (1) Laura Alejandra Suárez Pinto (D) ${ }_{1}$, William Rojas ${ }^{1}{ }_{1}$ \\ ${ }^{1}$ Fundación Universitaria de Ciencias de la Salud, Bogotá, Colombia
}

Cómo citar: Arenas S, Amaya-Veronesi L, Nieto-Zambrano PD, Mendieta-Roa DE, Suárez-Pinto LA, Rojas W. Embolia pulmonar como hallazgo inicial de enfermedad de Graves. Rev. Colomb. Endocrinol. Diabet. Metab. 2021;8(2):e712. https://doi.org/10.53853/encr.8.2.712

\section{Resumen}

Introducción: la enfermedad de Graves se conoce como una de las patologías tiroideas de origen autoinmunitario más frecuentes a nivel mundial. Se caracteriza por un aumento en la producción de hormonas tiroideas y de factores de coagulación, lo que produce un síndrome clínico con componente autoinmune y metabólico, propio de la respuesta fisiológica al estímulo de la tiroxina, constituyendo un factor de riesgo para eventos trombóticos.

Se ha descrito la relación entre la función tiroidea y la hemostasis a nivel corporal demostrando que las altas concentraciones de hormona tiroidea se relacionan con un mayor riesgo de trombosis venosa.

Presentación del caso: se trata de una mujer con clínica y diagnóstico de neumonía adquirida en la comunidad, con antibiótico de terapia empírica, con evolución clínica estacionaria, de progresivo requerimiento de oxígeno suplementario, taquicardia persistente y dolor torácico de características pleuríticas. Se sospecha embolia pulmonar, confirmando diagnóstico con angiotomografía de tórax que corrobora el compromiso trombótico. Se hicieron tamizajes de trombofilias, encontrando incidentalmente una función tiroidea alterada, diagnosticando hipertiroidismo, por lo que se indicó la realización de una gammagrafía de tiroides en la cual se observó captación de radioisotopo siendo concordante con etiología autoinmune. Se documenta y realiza el diagnóstico de embolia pulmonar como hallazgo inicial de enfermedad de Graves.

Discusión y conclusión: la enfermedad tiroidea, particularmente la enfermedad de Graves, es subestimada como etiología dentro del abordaje de pacientes con trombofilia. La valoración de la función tiroidea dentro del abordaje de los pacientes con eventos tromboembólicos debe considerarse para favorecer un abordaje integral y una orientación terapéutica dirigida.

Palabras clave: enfermedades de la tiroides, hipertiroidismo, enfermedad de Graves, trombosis, embolia y trombosis, embolismo pulmonar.

అ Correspondencia: Sebastián Arenas, Cl. 10 \# 18-75, Servicio de Medicina Interna, Hospital de San José, Sociedad de Cirugías de Bogotá, Fundación Universitaria de Ciencias de la Salud (FUCS), Bogotá, Colombia.

Correo-e: semaldonado@fucsalud.edu.co 


\section{Pulmonary embolism as an initial finding of Graves' disease}

\section{Abstract}

Introduction: Graves' disease is known as one of the most frequent thyroid pathologies of autoimmune origin worldwide. It is characterized by an increase in the production of thyroid hormones and coagulation factors; which produces a clinical syndrome with an autoimmune and metabolic component. Characteristic of the physiological response to thyroxine stimulation, constituting a risk factor for thrombotic events.

The relationship between thyroid function and hemostasis at the body level has been described, showing that high concentrations of thyroid hormone are related to an increased risk of venous thrombosis.

Case presentation: This is a woman with symptoms and a diagnosis of community-acquired pneumonia with empirical antibiotic therapy, with stationary clinical course, progressive requirement for supplemental oxygen, persistent tachycardia and chest pain with pleuritic characteristics. Pulmonary embolism is suspected, confirming diagnosis with chest CT angiography that confirms thrombotic involvement. Screening for thrombophilia was performed, incidentally finding altered thyroid function diagnosing hyperthyroidism, for which a thyroid scintigraphy was indicated in which radioisotope uptake was observed, being consistent with autoimmune etiology. The diagnosis of pulmonary embolism is documented and made as the initial finding of Graves' disease.

Discussion and conclusion: Thyroid disease (particularly Graves' disease) is underestimated as an etiology in the management of patients with thrombophilia. The assessment of thyroid function within the approach of patients with thromboembolic events should be considered to favor a comprehensive approach and targeted therapeutic orientation.

Keywords: Thyroid Diseases, Hyperthyroidism, Graves Disease, Thrombosis, Embolism and Thrombosis, Pulmonary Embolism.

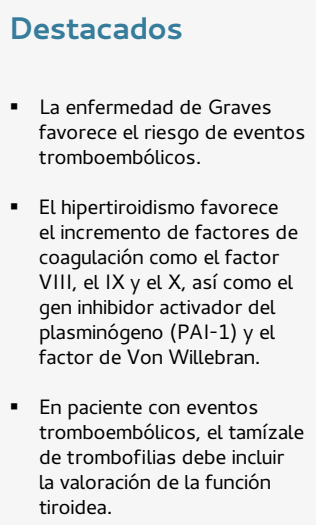

- El hipertiroidismo favorece el incremento de factores de coagulación como el factor VIII, el IX y el X, así como el gen inhibidor activador del plasminógeno (PAl-1) y el factor de Von Willebran.

- En paciente con eventos tromboembólicos, el tamízale de trombofilias debe incluir la valoración de la función tiroidea.

\section{Introducción}

La enfermedad de Graves es la patología autoinmune órgano-específica más común, denominada así en honor al doctor Robert James Graves, cirujano irlandés que la describió por primera vez en 1835. Esta se caracteriza por hipertiroidismo, bocio difuso, oftalmopatía y dermatopatía (mixedema pretibial), relacionando ocasionalmente estados variables de hipercoagulabilidad (1).

Esta enfermedad tiene una incidencia másalta en mujeres queenhombres, conuna relación de $10: 1$ y su presentación es más predominante entre la segunda y la quinta década de la vida. Las manifestaciones clínicas más comunes son nerviosismo, fatiga, palpitaciones, intolerancia al calor, pérdida de peso, taquicardia, tremor distal, debilidad muscular (>50\%), entre otros, los cuales (todos) son causados por el exceso de hormonas tiroideas y la actividad beta-adrenérgica incrementada. Los hallazgos de laboratorio son niveles suprimidos de TSH y niveles de T3 y T4 incrementados (1). 
El hipertiroidismo es el hallazgo más común, el cual es causado directamente por anticuerpos dirigidos contra el receptor tirotropina (anti-TSHR), el cual induce la activación permanente del receptor y que resulta en estimulación, síntesis y secreción de hormonas tiroideas $(2,3)$.

En el hipertiroidismo se ha observado un estado de hipercoagulabilidad e hipofibrinólisis con aumento de los factores de coagulación VIII, IX, X y del gen inhibidor activador del plasminógeno (PAI-1) (4). Estos niveles de factores aumentan en relación a las crecientes concentraciones de hormonas tiroideas, lo que constituye un factor de riesgo para eventos trombóticos (5).

La asociación entre el sistema hemostático y las enfermedades tiroideas se conoce desde el año de 1913, cuando Kaliebe informó acerca de un episodio de trombosis venosa cerebral en un paciente con tirotoxicosis (6); sin embargo y a pesar de que se han documentado varias alteraciones en los sistemas de coagulación que conducen a un estado de hipercoagulabilidad en pacientes con hipertiroidismo, no se han logrado dilucidar completamente las vías y los mecanismos patogénicos exactos para explicar esta asociación $(5,7)$.

A lo largo del tiempo se han descrito diversas anomalías adquiridas de la coagulación y la fibrinólisis en pacientes con disfunción tiroidea. Estas varían desde alteraciones subclínicas de laboratorio hasta hemorragias mayores en pacientes con hipotiroidismo manifiesto o eventos tromboembólicos fatales en pacientes hipertiroideos, con un aumento de la mortalidad a largo plazo por enfermedades cardiovasculares y cerebrovasculares (8), ello representa el $18 \%$ de las muertes asociadas con tirotoxicosis (9), donde se ha logrado demostrar que las hormonas tiroideas cumplen una función importante en la modulación de la hemostasia (10).

A través de numerosos estudios se ha logrado evidenciar la presencia de eventos trombóticos (como la trombosis venosa profunda, TVP) en pacientes con hipertiroidismo sintomático, en los cuales se desencadenó un estado de hipercoagulabilidad debido a niveles elevados del factor VIII y del factor von Willebrand, la disfunción endotelial y el aumento de la adhesión plaquetaria $(8,9)$.

A continuación se presenta el caso de una paciente que debuta con tromboembolismo pulmonar no provocado y una posible asociación con hipertiroidismo de novo.

\section{Presentación del caso}

Se trata de una paciente de sexo femenino de 64 años, quien ingresa al servicio de urgencias refiriendo un cuadro clínico de ocho días de evolución consistente en dolor torácico de características pleuríticas, asociado a disnea progresiva hasta presentarse incluso en reposo. Adicionalmente, tiene múltiples accesos de tos con movilización de secreciones de aspecto purulento, ocasionalmente hemoptoico y fiebre cuantificada en $38,5^{\circ} \mathrm{C}$.

Como antecedentes refirió hipertensión arterial y asma, por lo que recibía manejo con losartán, salbutamol y bromuro de ipratropio a necesidad. 
A la revisión por sistemas comentó que tuvo pérdida no intencional de 12 kilos en los últimos dos meses, palpitaciones ocasionales, cuadros intermitentes de deposiciones de consistencia líquida y episodios inexplicables de diaforesis.

Al examen físico de ingreso se encuentra una paciente normotensa, taquicárdica, con taquipnea y en la auscultación se encontró una disminución de ruidos respiratorios a nivel de base pulmonar derecha, con estertores de burbuja fina en campos medios y sin signos de bronco-obstrucción.

En el servicio de urgencias se le realiza un electrocardiograma que documenta la presencia de taquicardia supraventricular, para lo cual fue manejada con dosis única de betabloqueador oral con posterior mejoría en frecuencia cardiaca.

Se realizan paraclínicos de extensión sin evidencia de respuesta leucocitaria, sin anemia, ni irregularidades en el conteo plaquetario, destacando una importante cayademia (34\%) en el hemograma, con parámetros de función renal y electrolitos normales, gases arteriales en equilibrio de ácido base pero con hipoxemia (PO2 $55 \mathrm{~mm} \mathrm{Hg}$ ). Los Rx de tórax de ingreso (figura 1) mostraban como hallazgos de relevancia una radio-opacidad a nivel basal derecha, por lo cual y teniendo en cuenta el cuadro clínico referido, hacen suponer un posible cuadro consolidativo de etiología infecciosa para el cual se inicia antibioticoterapia empírica.

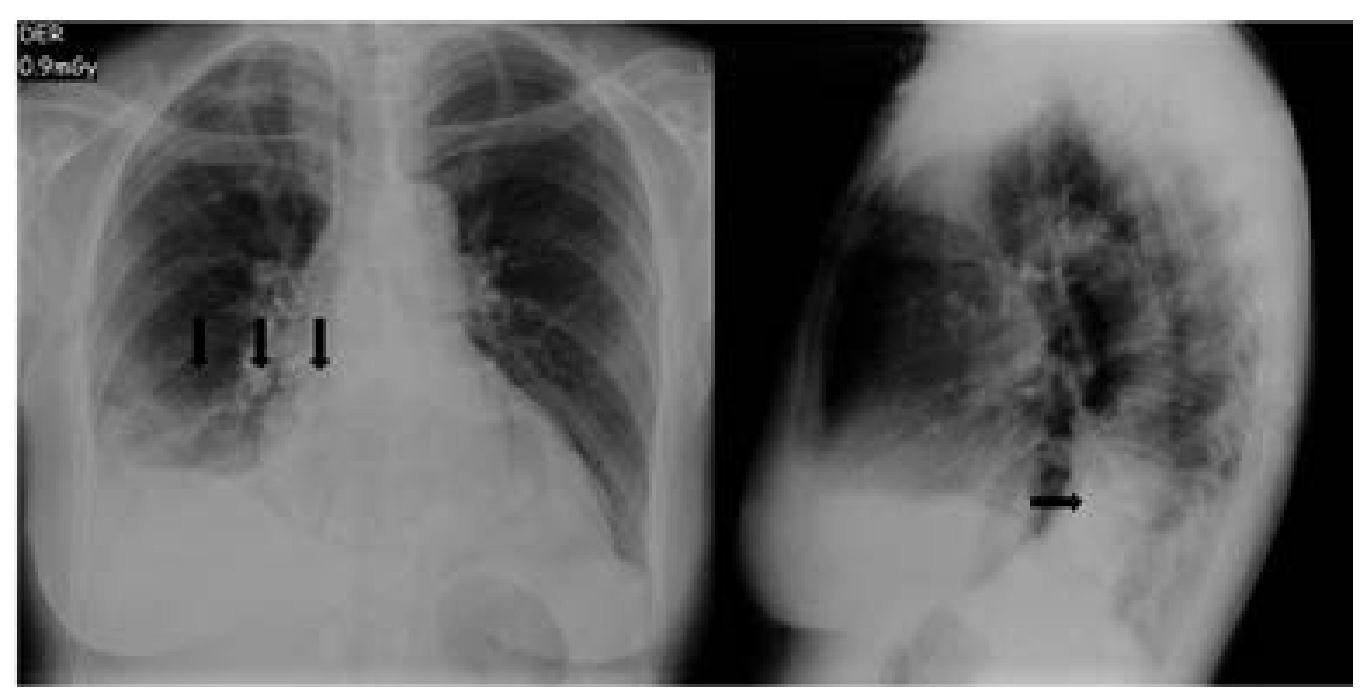

Figura 1. Rx de tórax en proyecciones PA y lateral

Nota explicativa de la imagen: se destaca la pérdida de ángulo costo-diafragmático derecho en relación con radio-opacidad que compromete el lóbulo inferior derecho, la cual igualmente genera pérdida de la radio-lucidez retrocardiaca.

Fuente: elaboración propia. 
Posterior al comienzo del manejo médico, se observó una evolución clínica estacionaria con aumento de los requerimientos de $\mathrm{O} 2$ suplementario hasta requerir un alto flujo mediante el sistema Ventury al $50 \%$, con taquicardia persistente y dolor pleurítico, por lo que se plantea como posibilidad diagnóstica una embolia pulmonar aguda, razón por la que realiza angiotomografía de tórax (angio TAC), el cual corrobora el compromiso trombótico sobre la rama lobar derecha y de las segmentarias en el lóbulo inferior derecho (figuras 2).
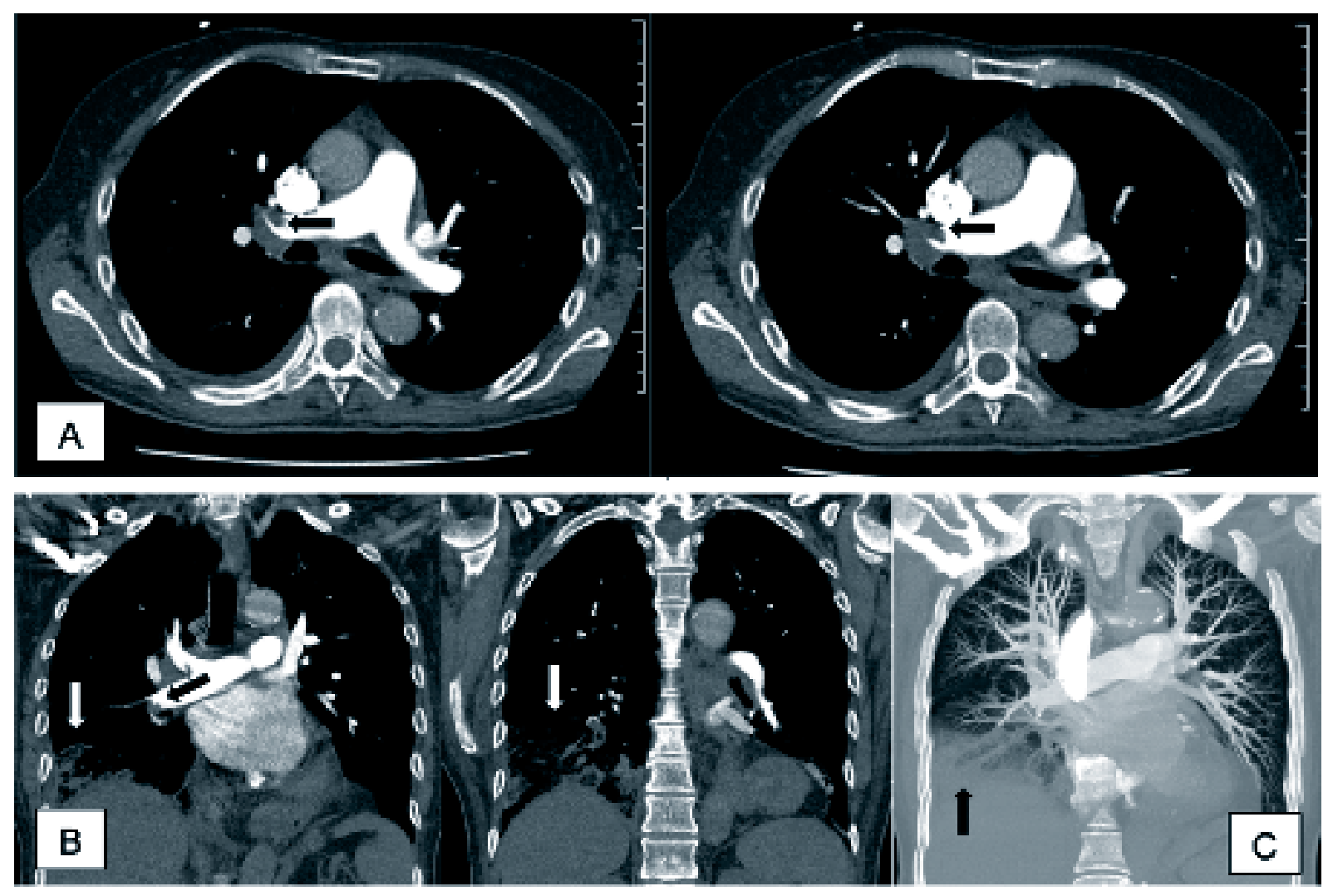

Figuras 2. AngioTAC de vasos de tórax

Nota explicativa por imagen: $(A)$ ventana angiográfica horizontal donde se observa el compromiso trombótico de la arteria pulmonar derecha; (B) ventana angiográfica frontal donde se observa la presencia de material trombótico que ocluye el flujo de la arteria pulmonar derecha y de su rama al lóbulo inferior derecho, además de infarto pulmonar en segmento posterior de lóbulo inferior derecho; $(C)$ ventana de vasos pulmonares donde se observa el defecto de paso de medio de contraste a vasos pulmonares de lóbulo inferior derecho.

Fuente: elaboración propia. 
Posterior a la confirmación del diagnóstico de embolismo pulmonar, se inicia el manejo de anticoagulante a dosis plena y se amplían los estudios en búsqueda de etiología maligna o autoinmune mediante ANAs, ENAs, $\beta 2$ Glicoproteina (IgM e IgG), anticuerpos anticardiolipinas y anticoagulante lúpico, resultando negativos. Adicionalmente, se corroboró la ausencia de lesiones sugestivas de neoplasia en estudios como TAC de cuello, tórax y abdomen e igualmente se solicitó un perfil metabólico, en donde llama la atención la presencia de TSH suprimida $(<0,015$ $\mathrm{uUl} / \mathrm{mL}$ ), razón por la que se obtiene una muestra para procesamiento de $T 4 L$, la cual se encuentra elevada $(64,3$ $\mathrm{pmol} / \mathrm{L}$ ) y confirmando de esta manera el diagnóstico de hipertiroidismo.

La paciente es valorada por el servicio de Endocrinología, quienes solicitan una gammagrafía de tiroides que muestra una importante captación de radioisótopo (figura 3), lo cual es concordante con una etiología autoinmune (enfermedad de Graves).
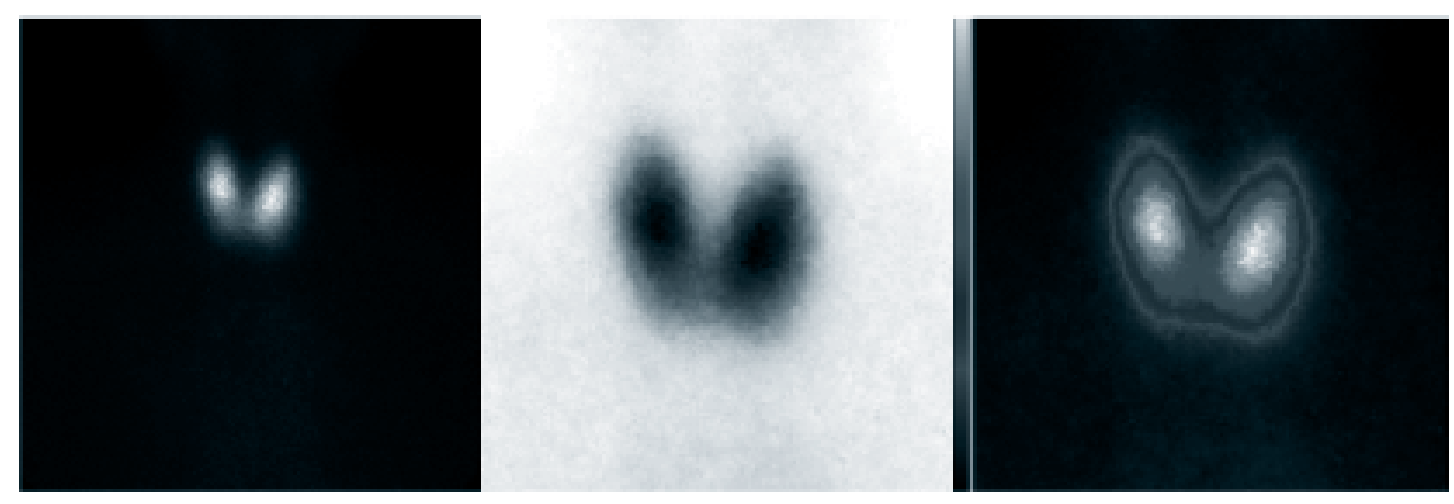

Figura 3. Gammagrafía nuclear de tiroides

Nota explicativa de la imagen: se observa un aumento de tamaño, una captación uniforme del radiofármaco y los hallazgos compatibles con bocio hipercaptante.

Fuente: elaboración propia.

Posteriormente, se da inicio de terapia antitiroidea con metimazol, confirmando una progresiva reducción de $\mathrm{T} 4 \mathrm{~L}(45,9$ y $40,6 \mathrm{pmol} / \mathrm{L})$. Igualmente, se realiza una medición de anticuerpos antirreceptores de TSH, los cuales se encontraron elevados (1,6 UI/L), favoreciendo el diagnóstico de enfermedad de Graves. Desde el punto de vista clínico, la paciente progresivamente presenta una estabilización de sus constantes vitales: paulatina normalización de cifras de frecuencia cardiaca, así como adecuada tolerancia a la reducción de aporte de oxígeno, alcanzando metas de $\mathrm{SaO} 2$ al medioambiente con remisión de accesos de tos, así como de la expectoración.

Al presentar una mejoría de su sintomatología, la paciente egresa de la institución a su domicilio, continuando controles con el servicio de Endocrinología ambulatoria y terapia antitiroidea. 


\section{Discusión}

Las patologías tiroideas tienen un amplio y variado espectro de presentación clínica, esto como resultado de la falta o el exceso en la función de la tiroxina, involucrada en un importante número de vías metabólicas para el funcionamiento de todo el organismo, incluyendo la homeostasis sanguínea.

El hipertiroidismo se ha relacionado con alteraciones hemostáticas que predisponen a estados variables de hipercoagulabilidad (6). Esto se ha demostrado por un aumento de los niveles plasmáticos del factor de von Willebrand (FVW) en pacientes con TSH suprimida y una función plaquetaria mejorada en comparación con pacientes eutiroideos (8). De igual forma, se ha reportado un incremento en los niveles de los factores de coagulación II, VII y $X(11)$.

En el estudio de Koster et al. (12) se reportó que los pacientes con hipertiroidismo tenían niveles aumentados de fibrinógeno plasmático, factor IX, FVW, antitrombina e inhibidor del activador del plasminógeno (PAI -1), junto con niveles reducidos de factor $X$ y activador del plasminógeno tisular (t-PA), lo que sugiere una actividad fibrinolítica plasmática globalmente reducida.

Los factores de riesgo que predisponen a eventos procoagulantes se relacionan con la triada de Virchow, pues se ha reportado que los pacientes con hipertiroidismo a menudo pueden presentar disfunción endotelial, disminución de la actividad fibrinolítica y estados de hipercoagulación que contribuyen al desarrollo de trombosis venosa y un mayor riesgo de embolia pulmonar (13).

No obstante, es de resaltar que la disfunción tiroidea, pese a relacionarse con alteraciones de la coagulación sanguínea, no es la causa más frecuente dentro del estudio de una trombofilia, razón por la cual debe considerarse dentro de los factores de exclusión al momento de orientar un abordaje diagnóstico de una coagulopatía. Como lo muestra el caso, la paciente mostraba signos y síntomas que exponían un cuadro constitucional crónico que obligaba a descartar otras causas, las cuales se vieron mimetizadas por la disfunción tiroidea que se diagnosticó.

Está bien establecido que la actividad del factor VIII de coagulación plasmática se correlaciona positivamente con la tasa metabólica tisular y los niveles de catecolaminas plasmáticas. Por su parte, una actividad adrenérgica excesiva, que ocurre en pacientes con hipertiroidismo, puede contribuir directamente a un aumento de la producción de factor VIII y en algunos estudios se ha descrito que el propanolol puede inhibir el aumento de FVIII en pacientes con hipertiroidismo (14).

En este sentido, se podría proponer que un peor control en la función tiroidea deriva en un mayor riesgo de eventos trombóticos. En el estudio de Rogers y colaboradores se informó que 21 de 22 pacientes con hipertiroidismo no tratados tenían niveles aumentados de actividad del factor VIII plasmático (FVIII: C), antígeno del FvW y cofactor de ristocetina (FvW: Rco), lo cual se normalizó después del tratamiento farmacológico con antitiroideos (15). 
Cabe anotar que la anticoagulación sigue siendo el pilar del tratamiento en cualquier condición trombótica independiente de su localización. La elección de la terapia anticoagulante debe hacerse de manera individualizada, particularmente a la condición de cada paciente. Hasta la fecha, en hipertiroidismo no se conoce una recomendación específica para la prescripción o el manejo anticoagulantes, sin embargo, son de tener en cuenta las interacciones farmacoterapéuticas que pueden tener estos medicamentos con los fármacos antitiroideos, ya que pueden tener una reducción en sus concentraciones plasmáticas (como en el caso de la warfarina), es por tal motivo que las terapias con heparinas de bajo peso molecular o fondaparinux siguen siendo de elección por su baja interacción farmacológica $(2,3,6)$.

De esta forma, es demostrada la relación entre eventos trombóticos e hipertiroidismo. Por su parte, la trombosis venosa profunda es el evento trombótico más frecuentemente reportado, así como trombosis en venas cerebrales, aunque en el estudio de Franchini et al. se documentaron cuatro pacientes con embolia pulmonar no provocada, en probable relación con hipertiroidismo manifiesto (3). En otro estudio, el riesgo de tener embolia pulmonar durante un periodo de seguimiento a cinco años fue 2,31 veces mayor para los pacientes con hipertiroidismo que para los pacientes eutiroideos (16).

En Latinoamérica se cuenta con dos reportes: una revisión en donde Palma et al. describen un caso de trombosis en senos venosos cerebrales relacionado con hipertiroidismo y enfermedad de Graves (17) y otro donde Benavides et al. describen el caso de un paciente con trombosis venosa profunda que muestra hipertiroidismo como único factor predisponente (9). En nuestro conocimiento, el presente caso es el primero que se reporta entre enfermedad de Graves y embolia pulmonar.

Se hace fundamental reconocer al hipertiroidismo y a la enfermedad de Graves como predisponentes de alteración en la homeostasis sanguínea. El reconocimiento de esta patología dentro del abordaje y estudio de cualquier evento trombótico resulta fundamental para una adecuada orientación diagnóstica y terapéutica, por lo que se deben invertir mayores esfuerzos en investigación para objetivar y estandarizar su orientación diagnóstica, así como establecer las mejores indicaciones en intervención, tiempo de esta y seguimiento de estos pacientes.

\section{Conclusiones}

El abordaje del paciente con trombofilia debe ser integral; debe incluir una valoración complementaria del estado metabólico del individuo para reafirmar la adecuada funcionalidad de la tiroides. El reconocimiento de la enfermedad de Graves como una patología con un potencial trombofílico permitiría más diagnósticos oportunos y terapias de manejo precoces, con un impacto sobre el pronóstico tanto de la enfermedad tiroidea como del evento tromboembólico que con ella se relaciona. 


\section{Agradecimientos}

A los doctores Daniela Jurado y Juan Nofal, quienes fueron partícipes en el diagnóstico, evolución y seguimiento del caso de la paciente, así como al profesor John Jaime Sprockel, instructor del Servicio de Medicina Interna de la Fundación Universitaria de Ciencias de la Salud y el Hospital de San José - Sociedad de Cirugías de Bogotá, quien fue líder en la conducción y el direccionamiento de este.

\section{Declaración de fuentes de financiación}

El presente estudio no tuvo financiación.

\section{Conflictos de interés}

Los autores declaran que no tienen conflictos de interés.

\section{Referencias}

[1] Cieza-Diaz D, Centeno-Clemente C, Pinto-Valdivia M. Características clínicas, perfil hormonal y marcadores de autoinmunidad de pacientes con enfermedad de Graves. Rev Med Hered. 2008;19(4):152-7. https://doi. org/ 10.20453/rmh.v 19i4.957

[2] Elbers LPB, Squizzato A, Gerdes VEA. Thyroid disorders and hemostasis. Semin Thromb Hemost. 2018;44(7):676-82. https://doi. org/10.1055/s-0038-1666825

[3] Franchini M. Hemostatic changes in thyroid diseases: haemostasis and thrombosis. Hematology. 2006;11(3):203-8. https://doi. org/ 10.1080/ 10245330600667591

[4] van Zaane B, Stuijver DJF, Squizzato A, Gerdes VEA. Arterial and venous thrombosis in endocrine diseases. Semin Thromb Hemost. 2013;39(5):489-95. https://doi. org/ 10.1055/s-0033-1343889

[5] Debeij J, Dekkers OM, Asvold BO, Christiansen SC, Naess IA, Hammerstrom J, et al. Increased levels of free thyroxine and risk of venous thrombosis in a large population-based prospective study. J Thromb Haemost. 2012;10(8):1539-46. https:// doi .org/ 10.1111/ j.15387836.2012.04818.x

[6] Squizzato A, Romualdi E, Büller HR, Gerdes VEA. Clinical review: Thyroid dysfunction and effects on coagulation and fibrinolysis: a systematic review. J Clin Endocrinol Metab. 2007;92(7):2415-20. https:// doi.org/ 10.1210/j c.20070199

[7] Franchini M, Lippi G, Targher G. Hyperthyroidism and venous thrombosis: a casual or causal association? A systematic literature review. Clin Appl Thromb Hemost. 2011;17(4):387-92. https://doi. org/ 10.1177/ 1076029610364521

[8] Homoncik M, Gessl A, Ferlitsch A, Jilma B, Vierhapper H. 
Altered platelet plug formation in hyperthyroidism and hypothyroidism. J Clin Endocrinol Metab. 2007;92(8):3006-12. https:// doi.org/10.1210/ jc.20062644

[9] Benavides J, Vivas J, Jaramillo G, Berna L. Trombosis venosa profunda en un paciente con hipertiroidismo de novo. Presentación de caso. Rev Repert Med y Cirugía. 2017;26(1):44-9. https://doi org/ 10.1016/j.reper.2017.03.004

[10] Rodilla-Fiz A, Garví-López M, Gómez-Garrido M, Girón-la-Casa M. Alteraciones de la hemostasia y trombosis asociadas a enfermedad tiroidea, a propósito de 2 casos. Rev Esp Anestesiol Reanim. 2015;63(6):357-60. https://doi . org/ 10.1016/j.redar.2015.10.004

[11] Loeliger EA, van-Der-Esch B, Mattern $M$, Hemker $H$. The biological disappearance rate of prothrombin, factors VII, IX and $X$ from plasma in hypothyroidism, hyperthyroidism, and during fever. Thromb Diath Haemorrh. 1964;1(10):267-77. https://doi. org/ 10.1055/s-0038-1654782

[12] Koster T, Blann A, Briët E, Vandenbroucke J, Rosendaal F. Role of clotting factor VIII in effect of von Willebrand factor on occurrence of deep-vein thrombosis. Lancet. 1995;345(8943):152-5. https:// doi.org/ 10.1016/ S01406736(95)90166-3

[13] Grine S, Charfi N, Kamoun M, Mnif F, Ben-Naceur B, Rekik N, et al. Hyperthyroidism: A rare cause of pulmonary embolism: Report of two cases. Indian J Endocrinol Metab. 2013; 17(6):1104-7. https://doi. org/ 10.4103/2230-8210.122640

[14] Maes J, Michotte A, Velkeniers $B$, Stadnik T, Jochmans $K$. Hyperthyroidism with increased factor VIII procoagulant protein as a predisposing factor for cerebral venous thrombosis. J Neurol Neurosurg Psychiatry. 2002;73(4):458. https://doi. org/ 10.1136/jnnp.73.4.458

[15] Rogers J, Shane S, Jencks F. Factor VIII activity and thyroid function. Ann Intern Med. 1982;97(5):7136. https://doi.org/ 10.7326/00034819-97-5-713

[16] Lin H, Yang L, Kang J. Increased risk of pulmonary embolism among patients with hyperthyroidism: a 5-year follow-up study. J Thromb Haemost. 2010;8(10):2176-81. https:// doi .org/ 10.1111/ j . 15387836.2010.03993.x

[17] Palma DA, Popescu BM. Trombosis de senos venosos cerebrales e hipertiroidismo de Graves ¿un factor predisponente? Rev Fac Med Univ Nac Nord. 2004;22(1/2):16-8. 